\title{
ANALYSIS OF MODE CHOICE BEHAVIOR AND VALUE OF TIME IN DHAKA CITY, BANGLADESH
}

\author{
Faysal Ibna Rahman ${ }^{1}$, Md. Mahabubul Bari ${ }^{2}$, Md. Aminul Islam ${ }^{3}$, Tonmoy Paul Joyanto ${ }^{4}$ \\ ${ }^{1}$ University of Yamanashi, Faculty of Civil and Environmental Engineering, Japan \\ ${ }^{2}$ Kathahira \& Engineers International, Japan \\ ${ }_{1,3}^{1, N i p p o n ~ K o e i ~ B a n g l a d e s h ~ L t d ., ~ B a n g l a d e s h ~}$ \\ ${ }^{4}$ Development Design Consultant (DDC), Bangladesh
}

Received 21 October 2019; accepted 23 January 2020

\begin{abstract}
To make public transportation more efficient and user-friendly it is important to reveal the important factor affecting their mode choice decision and explain the trade-offs which they usually make while choosing the mode of transportation. Without addressing this aspect, there is every possibility of failure of the new strategies to be taken. In this study, mode choice behavior and value of time of Dhaka city dwellers are calculated considering income level, trip purpose and trip length using RP and SP data. In mode choice analysis, we consider a total of ten modes among those eight modes are already existing and other two modes (MRT and BRT) are up-coming. The multinomial logit model is used for mode choice analysis and the binary logit model is used for the value of time estimation. Study indicates that people prefer to ride newly build MRT and BRT. Change of mode choice is also found with the change of trip purpose, trip length and income level of trip makers. Waiting time shows always higher value than in-vehicle time.
\end{abstract}

Keywords: modal split, SP survey, transport planning, value of time.

\section{Introduction}

The mobility of the city dwellers is increased radically due to the rapid progress of urbanization and growth in population in the city area. Consequently, travel demand among the city dwellers has also increased proportionally. Hence it is the prime object of transport planners to coordinate transportation supply with increasing travel demand. To accomplish this target travel demand should be assessed precisely. Travel demand estimation is a four-stage process that involves trip generation, trip distribution, mode choice and traffic assignment out of which mode choice analysis plays a vital role as it deals with predicting mode used by the travelers to reach their destination. Many factors are responsible for the mode choice decision. A proper analysis of mode choice decision can help in addressing disputes such as forecasting demand mode for new modes of transportation, mitigating traffic congestion, allocating resources, examining the general efficiency of travel and will provide insight into traveler's behavior characteristics.

Dhaka is one of the world's largest and fastest-growing cities faces acute transport problems. From 3 million in 1971, its metropolitan population has grown

\footnotetext{
${ }^{1}$ Corresponding author: aminul-is@nkbangladesh.co.bd
} 
exponentially to around 18 million todays. Dhaka has also become the world's most crowded large city, with double or treble the population density of other world cities such as Tokyo, London, and shanghai. Even Mumbai and Kolkata have less than twothirds of Dhaka's density. Moreover, Dhaka is expected to grow to over 26 million by 2035. A huge population is expected to generate a commensurable number of trips per day. To reduce traffic jams and meet excessive travel demand, in 2015 an updated transport strategy for the city was prepared for looking at transport infrastructure and management proposals for the next 20 years (2015-2035). The RSTP (Revised Strategy Transport Plan) proposed five metro-rail lines (Mass Rapid Transit- MRT), two Bus Rapid Transit lines (BRT) (RSTP, 2016).

\section{Literature Review}

The first initiative to develop the mode choice model for Dhaka was the Greater Dhaka Metropolitan Area Integrated Transport Study (DITS, 1993). It was intended to evaluate the long-term travel demand of Dhaka applying the traditional four-step model. The model considered walk, rickshaw, public transport, and private transport modes and data were collected from the Household Interview Survey (HIS). It was simplified into the binary logit model. However, since not all of these modes are not competing for modes of each other, the estimation could not produce a realistic result. The model was estimated that $60 \%$ of the trips are walking which are unrealistically high trips. Again, the bus and tempo were both considered as public transport while taxi and car both considered private transport modes. This generalization could not explore the diversity of the mode choice of travelers. Strategic Transport Plan (STP) developed a mode choice for Dhaka as a part of the travel demand model forecasting model in 2005 (STP, 2005). The model considered only two types of modetransit and non-transit motorized modes were considered. But walking and NonMotorized Transport were not included in the model although a considerable number of people in Dhaka use those modes. Only travel time and cost were considered as mode choice variables although socio-demographic characteristics of the travelers and other attributes of the transport modes influence the travel behavior. Enam (2010) pointed out the inconsistency of STP model estimation as the value of time of low-income and high-income groups gave counterintuitive results. Hasan (2007) also studied the mode choice behavior according to the trip purpose (home base work trip, home base education trip, home base other trip and non-home base trip). Besides, it adopted a rule base approach for model development. The decision followed in such a way that travelers with car ownership will always choose a car for traveling and others will choose from the rickshaw, auto-rickshaw, and transit. Mode choice behavior among different alternatives was studied using the Multinomial Logit (MNL) model.

Enam (2010) studied the mode choice behavior of travelers to capture the preference for the proposed BRT and MRT. The mode choice model was developing combining SP and RP data. The study incorporating socio-economic variables like income, age, gender, occupation, employment, etc. into the model. The value of time of different modes was calculated and compared with the previous studies. In the first step, the modal split has been 
made among walking, rickshaw and other modes (which includes private car, bus, and auto-rickshaw) using the shortest path distance as the only explanatory variable. In the second step, other modes have divided into a private car, bus, and auto-rickshaw using respective travel costs as the only explanatory variable. However, such a generalized model cannot predict the actual behavior as it did not consider the taste heterogeneity in terms of other factors that are likely to affect the choice of modes.

\section{Objectives}

This study is done to overcome the previous study and capture the perfect scenario of mode choice of Dhaka city. State Preference (SP) survey is conducted to analyze the mode choice behaviors for Dhaka metro city dwellers considering existing eight types mode (walking, rickshaw, private car, private motorcycle, tri-motorized cycle, public bus, human hauler, paratransit) and upcoming two modes (MRT, BRT). To capture the full market segment, sample data are collected considering income level (high, medium, and low income) and trip purpose (business, commuting, and others) for short, medium and long-distance trips. Mode choice attributes such as waiting time, in-vehicle time, cost and seating option are considered in this analysis. This study is done into two parts:

- Multinomial Logit (MNL) model is used to mode choice behavior according to the combination of income level, trip purpose, and distance;

- Binary Logit Model is used to estimate the value of time and value of waiting time considering the combination of income level, trip purpose.

This study is done to revel the mode choice preference for policymakers to take suitable steps in future transportation planning.

\section{Study Area}

The survey was carried in selected areas of Dhaka where stations of MRT Line - 1 are planned to be located. Data are collected from thirteen locations along the main corridor (Kamlapur to Airport) of MRT Line -1. From Airport to Kamlapur there is a total 78 number of segments out of 25 segments are Short Trips, 16 segments are medium trips and 37 segments are Long Trips. Trip type (long, medium and short trip) are classified according to the length shown in Table 1 . The survey corridor is shown in Figure 1.

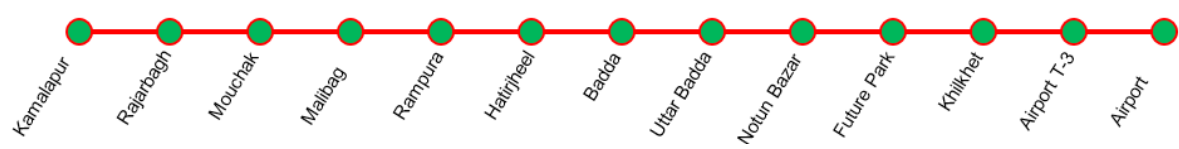

Fig. 1.

Survey Corridor (Kamlapur to Airport) of MRT Line 1 
Table 1

Classification of Trip Type According to Distance

\begin{tabular}{|c|c|}
\hline Trip Type & Length $(\mathbf{k m})$ \\
\hline Short Trip & $1 \mathrm{~km} \sim 3.16$ \\
\hline Medium Trip & $3.17 \mathrm{~km} \sim 5.2$ \\
\hline Long Trip & More than $5.2 \mathrm{~km}$ \\
\hline
\end{tabular}

\section{Individual Mode Choice Behavior and Decision Rule}

The mode choice studies in transportation study can be broadly distinguished in terms of aggregate and disaggregate approach. In the aggregate approach, the modal split is straightforwardly assessed with data aggregated at the zonal level or for a group of travelers. Aggregate models endeavor to express to the normal conduct of a group of travelers rather than a solitary person. On the other hand, Individual mode choice behavior and decision rule are particularly important in the context of disaggregate modeling. As a result, it can reflect changes in choice behavior due to a change in individual characteristics and attributes of alternatives (Koppelman and Bhat, 2006).

In disaggregate approach, the unit of decision making is an individual traveler. An important requirement for modeling the mode choice is that the model should be guided by the behavioral principle of the travelers. In other words, it should represent the decision that travelers make when confronted with a choice. According to BenAkiva and Lerman (1985), the mode choice of travelers is the outcome of a sequential decision-making process, such as:

- Definition of Choice Problem;

- Generation of Alternatives;

- Evaluation of Attributes of Alternatives;

- Choice;

- Implementation.
The first step of the process is the definition of the choice problem. This is defined by the context of the trip, for example, mode choice for travel to work. Next, the traveler has to decide from the alternative modes. The mode which the traveler will take into consideration is largely determined by the availability of the modes, affordability of the travelers, informational constraints and so on. The traveler has to then evaluate the attributes (for example- time, cost, comfort, etc.) among the competing modes. To decide the alternative modes by evaluating the attributes, the traveler has to apply the decision rule.

The decision rule is important because it determines the ultimate choice. Even for the same set of alternatives and the same value of attributes, the choice can be different for different decision rules. A wide variety of decision rules are studied in decision theory literature (Svenson, 1979). Among all of the decision rules, the widest decision rule is the utility maximization rule which represents the rational choice-making of the travelers (Ben-Akiva \& Lerman, 1985). Under utility maximization rule, the utility of a travel mode is expressed as a function in terms of a combination of the attributes (for exampletravel time, cost, comfort, etc.). The traveler will choose the mode with the highest utility.

The discrete choice model is a mathematical function that predicts an individual's choice base on utility. Hence, the use of the binary 
logit model and multinomial logit model under discrete choice methods was an analytically convenient modeling method. The binary logit model is used when the individual has to choose in between two discrete alternatives. When more than two possible outcomes, the multinomial logit model is used (Eq. (1)).

$P_{n}(i)=\frac{e^{v_{i n}}}{\sum_{j=1}^{k} e^{v_{j n}}}$

Where,

$P_{n}(i)=$ Probability of individual $n$ choosing mode, $i$;

$v_{j n}=$ Utility derived by individual $\mathrm{n}$ from mode j;

$\mathrm{K}=$ Number of available modes of transportation.

The utility by an individual $\mathrm{n}$ from mode $\mathrm{j}, v_{j n}$, is derived as linear function as the explanatory variable as follows, Eq.(2):

$v_{j n}=B_{0 j}+B_{1 j} X_{1 n}+B_{2 j} X_{2 n}+\cdots+B_{n j} X_{q n}$

Where,

$B_{0 j}=$ Alternative Specific constant for mode j;

$B_{1 j}, B_{2 j}, \ldots \ldots, B_{n j}=$ Coefficients associated with explanatory variables;

$X_{1 n}, X_{2 n}, \ldots \ldots, X_{q n}=$ Explanatory variables for individual $\mathrm{n}$;

$\mathrm{q}=$ number of explanatory variables included in the model.

\section{Question Preparation and Model Specification}

The success of the State Preference (SP) survey depends on the context in which the survey questionnaire has been developed and the questions are asked. In this study, data are collected by the skilled person with realistic scenarios. Data are collected from a survey on 450 trip makers. 450 sets of questions are prepared considering income level, trip purpose, trip length, and origindestination location to capture the actual scenario in the study area. Each question set consists of an actual scenario with the best and worst condition according to attributes. The designing of the survey question of the mode choice model needs an extensive evaluation of observation of observed data and the efficiency of the whole mode system. In this study, mode choice utility is predicted based on specific parameters. Total ten types modes are considered for mode choice among those eight types mode (walking, rickshaw, private car, private motorcycle, tri-motorized cycle, public bus, human hauler, paratransit) are existing in present and two modes (MRT, BRT) are up-coming. Attributes of mode choice in this study are considered-waiting time, in-vehicle time, cost and seating option which are more prominent attributes to mode choice alternatives. Income level, trip purpose, and travel distance have a significant effect on mode choice with the attribute of mode alternatives (Ortuzar \& Willumsen, 2011). To capture the variation of social-economical variable and trip purpose and travel distance survey questions are prepared for data collection. Data collection considering the market segment (income level, trip purpose, trip length) also reduce the biases of result during data collection time to represent the actual mode choice among the study group. The trip purpose is divided into three typesbusiness trip (BT), commuting trip (CT), and other trip (OT). The commuting trio can be defined as the periodically recurring trip between one's place of residence and place of work or study and in doing exceed the boundary of their residential place. A business trip is a trip undertaken for work 
or business purposes. Remaining trips are then considered as other trips such as leisure trips, social trips, etc. Income level is also classified into three groups according to monthly income in BDT, shown in Table 2. In this survey, 27 types of decision-maker sets are created considering income level, trip purpose and trip length.

Table 2

Classification of Income Level According to Monthly Income

\begin{tabular}{|c|c|}
\hline Income Group & Income Range (in BDT) \\
\hline High Income (HI) & $>51,600$ \\
\hline Medium Income (MI) & $20,601 \sim 51,600$ \\
\hline Low Income (LI) & $<=20,600$ \\
\hline
\end{tabular}

To representative, the actual value of the attribute of each mode is also important to get an accurate decision. Before conducting the final survey, a pre-survey has been operated to get the representative value of attributes (waiting time, in-vehicle time, cost) of the existing eight modes for 78 numbers of the segment in the study area.

Table 3

Travel Cost for MRT and BRT

\begin{tabular}{|c|c|}
\hline Type & Fare (BDT) \\
\hline MRT & $22.6($ fixed $)+2.8 / \mathrm{km}$ \\
\hline BRT & $9.90($ fixed $)+4.5 / \mathrm{km}$ \\
\hline
\end{tabular}

Besides, based on the actual condition, the best and worst-case scenario has been developed for the alternatives depending on trip makers' income level and trip purpose. To develop the best and worst-case scenario for mode choice parameters, the boundary condition is considered, the upper level is high income business trip (HI-BT) and the lower level is low-income other trip (LI$\mathrm{OT})$. HI-BT prefers more willing to pay for less waiting and in-vehicle time. On the other hand, LI-OT prefers less willing to pay and not much sensitive to waiting and in-vehicle time. Within this boundary condition, other scenario parameters are assigned. In this study, it is assumed that HI-BT for best-case scenario will pay $50 \%$
A representative value of each modes is calculated from the mean value of taking five samples of each segment. The value of attributes of MRT and BRT are taken from the Preparatory Study on Dhaka Mass Rapid Transit Development Project (MRT Line-1) (FS MRT line-1), the cost is shown in Table 3. more than the actual cost and prefer $50 \%$ less waiting and in-vehicle time. It is further assumed the reverse scenario for LI-OT. The strategy of tuning parameters for best and worst scenarios considering income level and trip purpose are shown in Table 4. In the question paper, adding a best and worst condition with the actual scenario. We can get data about the importance of parameters in mode choice and willingness to pay for better service.

During the survey, the trip maker is first identified according to income level, trip purpose and trip length then trip maker make a multi-mode choice considering the given attributes for the actual, best and worst 
condition. Each question paper contains the actual, best and worst conditions. A trip maker can choose more than one mode among the given attribute. Considering actual, best and worst condition, a total of thirty hypothetical scenarios are presented in front of the trip maker to collect their preference data by taking "Yes" or "No". 450 number of question papers are prepared for Surveying on 450 number of the trip maker.
Among that $45 \%$ are considered for a short trip (ST), $25 \%$ are for medium trip (MT) and $30 \%$ are for the long trip (LT). To reduce biases in data collection. One-third of the sample is considered for high income (HI), one-third sample is considered for mediumincome (MI) and rest are considered for low income (LI). The same procedure is also applied for a business trip (BT), comminuting trip (CT), and other trip OT).

Table 4

Strategy of Attributes Tuning of Mode Considering Income Level and Trip Purpose

\begin{tabular}{|c|c|c|c|c|c|c|c|}
\hline \multirow{2}{*}{$\begin{array}{l}\text { Parameters } \\
\text { Low Income }\end{array}$} & \multicolumn{3}{|c|}{ Cost Changing } & \multirow{2}{*}{$\begin{array}{l}\text { Parameters } \\
\text { Low Income } \\
\end{array}$} & \multicolumn{3}{|c|}{$\begin{array}{l}\text { Waiting Time and In-Vehicle } \\
\text { Time Changing }\end{array}$} \\
\hline & Best & Actual & Worst & & Best & Actual & Worst \\
\hline Business & $35 \%$ & $0 \%$ & $-30 \%$ & Business & $-35 \%$ & $0 \%$ & $30 \%$ \\
\hline Commuting & $20 \%$ & $0 \%$ & $-40 \%$ & Commuting & $-20 \%$ & $0 \%$ & $40 \%$ \\
\hline Others & $15 \%$ & $0 \%$ & $-50 \%$ & Others & $-15 \%$ & $0 \%$ & $50 \%$ \\
\hline Medium Income & Best & Actual & Worst & Medium Income & Best & Actual & Worst \\
\hline Business & $40 \%$ & $0 \%$ & $-25 \%$ & Business & $-40 \%$ & $0 \%$ & $25 \%$ \\
\hline Commuting & $35 \%$ & $0 \%$ & $-30 \%$ & Commuting & $-35 \%$ & $0 \%$ & $30 \%$ \\
\hline Others & $25 \%$ & $0 \%$ & $-40 \%$ & Others & $-25 \%$ & $0 \%$ & $40 \%$ \\
\hline High Income & Best & Actual & Worst & High Income & Best & Actual & Worst \\
\hline Business & $50 \%$ & $0 \%$ & $-15 \%$ & Business & $-50 \%$ & $0 \%$ & $15 \%$ \\
\hline Commuting & $40 \%$ & $0 \%$ & $-20 \%$ & Commuting & $-40 \%$ & $0 \%$ & $20 \%$ \\
\hline Others & $30 \%$ & $0 \%$ & $-35 \%$ & Others & $-30 \%$ & $0 \%$ & $35 \%$ \\
\hline
\end{tabular}

\section{Mode Choice Analysis}

The multinomial logit model (MNL) is used for mode choice analysis as well as the impact of parameters on the mode choice decision. In the MNL model relative probability is estimated considering a reference mode. In this case, "rejection mode" is used as a reference. The coefficient of parameters and their significant are shown in Tables 5 and 6 respectively. If $p$-value less than 0.05 , Parameters have a significance impact on mode choice. There are two types of parameters such as co-variates (waiting time, in-vehicle time, cost) which have continuous value and other types are categorical values (trip length, trip purpose, income level, seating option). For each categorical variable, the last item has been taken as a reference to estimate the coefficient of the other variables. Therefore, reference variables coefficients are zero value. All the variables had significant parameter estimations and logical signs. The variable "travel cost" is expected to have a negative sign. As travel costs increase, the probability of selecting modes will decrease. A positive coefficient in the table means that the variables have a positive impact on utility and so reflects a higher probability of choosing the mode. The 
negative coefficient of variables indicates that with the increase of variable, the probability of selection of mode will be decreased.

In walking, waiting time and cost parameters are insignificant. With the increase in travel time, the choice of walking gradually decreases. For short and medium trip lengths, the rickshaw is more suitable than a long trip. For business purpose trips, the selection of rickshaw is decreased. In the case of private Motor-cycle waiting time and seating option attributes are insignificant. It is found that income and trip purpose play an important role in the mode choice decision for the private motorcycle. Waiting time for the tri-motorized cycle was unexpectedly positive, which means that the waiting time has less significance and not much influence as it does with other modes. Other variables of in-vehicle time, short trip length, and business and commuting trips have negative coefficients, which indicate the uses are less likely to choose a tri-motorized cycle in these situations.

Waiting time and Seating option is insignificant for private car users. The coefficient of the high-income level is positive, which implied that affluent users are more likely to use a private car than other modes where users are less sensitive to cost. As well, for short and medium-length trips private car is the preferable mode to choose. The estimated coefficients of business and commuting trips are negative, which indicates that it is more likely to choose private cars for other purposes. For public bus, the estimated coefficient for business and commuting is positive, which indicate that users are likely to choose public bus for these trips. Other variables coefficients are negative, implied that if these variables increase the probability to use the Public transport bus decrease.

\section{Table 5}

Coefficient Value of the Parameters

\begin{tabular}{|c|c|c|c|c|c|c|c|c|c|c|}
\hline 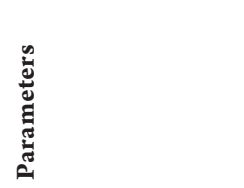 & 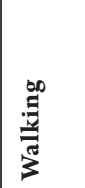 & 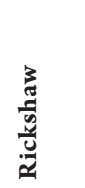 & 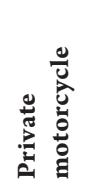 & 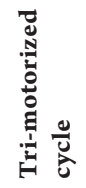 & $\begin{array}{l}\text { है } \\
\text { है } \\
\text { है } \\
\text { E }\end{array}$ & 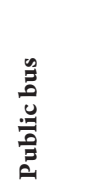 & 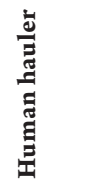 & 点 & 톰 & 苟 \\
\hline Intercept & 13.84 & -7.93 & 3.44 & -6.65 & -4.41 & 2.87 & 1.89 & 2.52 & 3.56 & -5.01 \\
\hline Waiting Time (min) & -2.16 & -0.18 & -10.50 & 0.26 & -11.21 & -0.03 & 0.13 & 0.41 & 0.10 & 0.31 \\
\hline In Vehicle time (min) & -0.16 & 0.04 & -0.01 & -0.30 & 0.01 & -0.01 & -0.02 & -0.29 & -0.14 & -0.05 \\
\hline Cost (BDT) & -0.02 & -0.02 & -0.09 & 0.11 & 0.01 & -0.11 & -0.12 & 0.01 & -0.02 & 0.01 \\
\hline Short trip & -3.45 & 5.68 & -2.25 & -0.28 & 1.78 & -2.42 & -2.94 & -7.16 & -4.95 & -1.25 \\
\hline Medium trip & -3.45 & 3.31 & -1.05 & 0.24 & 1.25 & -1.21 & -1.64 & -3.95 & -2.82 & -0.82 \\
\hline Long trip & 0.00 & 0.00 & 0.00 & 0.00 & 0.00 & 0.00 & 0.00 & 0.00 & 0.00 & 0.00 \\
\hline High income & -1.28 & 0.44 & 0.19 & 2.09 & 2.44 & -0.79 & -1.25 & 0.14 & -0.06 & 1.39 \\
\hline Medium income & -0.21 & 0.30 & 0.34 & 1.21 & 0.82 & -0.04 & -0.22 & 0.31 & 0.23 & 1.04 \\
\hline Low income & 0.00 & 0.00 & 0.00 & 0.00 & 0.00 & 0.00 & 0.00 & 0.00 & 0.00 & 0.00 \\
\hline Business trip & -0.29 & -0.33 & 0.46 & -0.49 & -0.34 & 0.21 & 0.16 & 0.16 & -0.02 & 0.16 \\
\hline Commuting trip & -0.47 & -0.13 & 0.66 & 0.95 & -0.23 & 0.26 & 0.07 & 0.18 & 0.08 & -0.29 \\
\hline Others & 0.00 & 0.00 & 0.00 & 0.00 & 0.00 & 0.00 & 0.00 & 0.00 & 0.00 & 0.00 \\
\hline Standing & 19.82 & -23.45 & -25.24 & -20.70 & -22.12 & -24.47 & -23.22 & 0.48 & 0.84 & -21.34 \\
\hline Seating & 0.00 & 0.00 & 0.00 & 0.00 & 0.00 & 0.00 & 0.00 & 0.00 & 0.00 & 0.00 \\
\hline
\end{tabular}




\section{Table 6}

Significant of Parameters (P-Value)

\begin{tabular}{|c|c|c|c|c|c|c|c|c|c|c|}
\hline 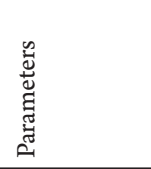 & 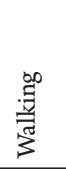 & 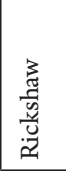 & 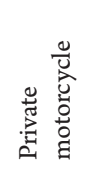 & 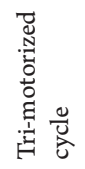 &  & 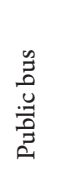 & 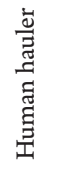 & 占 & 花 & 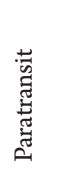 \\
\hline Intercept & .000 & .000 & .000 & .000 & .000 & .000 & .000 & .000 & .000 & .000 \\
\hline $\begin{array}{l}\text { Waiting } \\
\text { Time (min) }\end{array}$ & .985 & .000 & .940 & .000 & .948 & .004 & .000 & .000 & .000 & .000 \\
\hline $\begin{array}{l}\text { In Vehicle } \\
\text { time (min) }\end{array}$ & .000 & .000 & .022 & .000 & .133 & .006 & .000 & .000 & .000 & .000 \\
\hline Cost (BDT) & .999 & .000 & .000 & .000 & .000 & .000 & .000 & .613 & .000 & .000 \\
\hline Short trip & .000 & .000 & .000 & .404 & .000 & .000 & .000 & .000 & .000 & .000 \\
\hline Medium trip & .000 & .001 & .000 & .371 & .000 & .000 & .000 & .000 & .000 & .001 \\
\hline $\begin{array}{l}\text { High } \\
\text { income }\end{array}$ & .000 & .024 & .144 & .000 & .000 & .000 & .000 & .264 & .565 & .000 \\
\hline $\begin{array}{l}\text { Medium } \\
\text { income }\end{array}$ & .378 & .135 & .020 & .000 & .000 & .656 & .064 & .008 & .019 & .000 \\
\hline Business trip & .252 & .093 & .001 & .008 & .023 & .027 & .211 & .192 & .880 & .333 \\
\hline $\begin{array}{l}\text { Commuting } \\
\text { trip }\end{array}$ & .066 & .479 & .000 & .000 & .113 & .006 & .579 & .139 & .411 & .094 \\
\hline Standing & & .997 & .992 & .997 & .995 & .993 & .996 & .000 & .000 & .996 \\
\hline
\end{tabular}

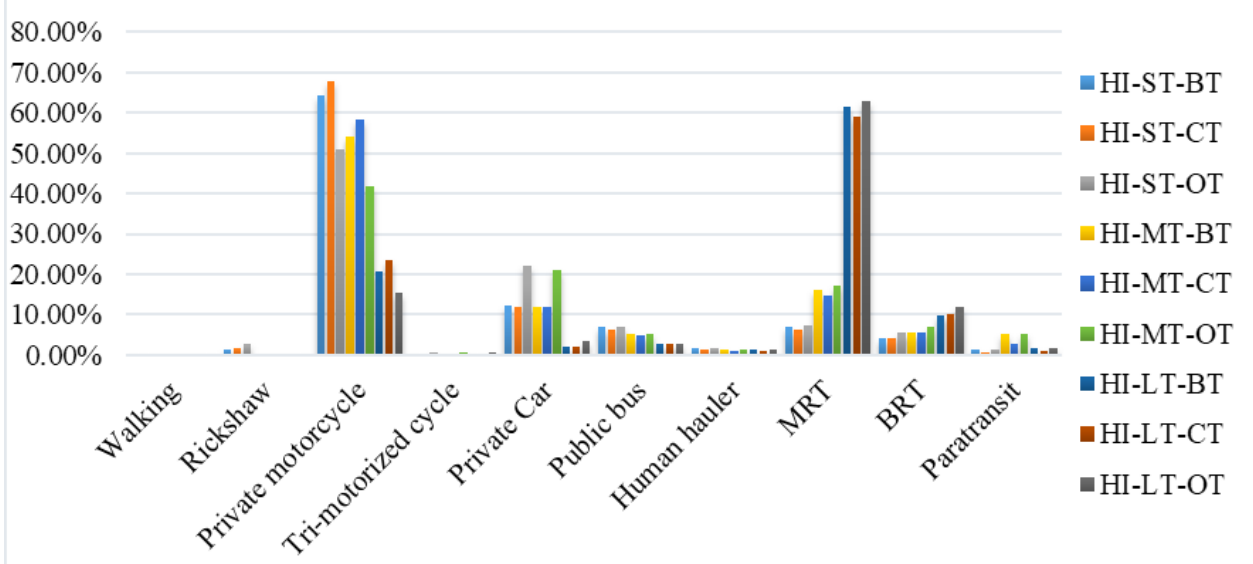

Fig. 2.

Mode Choice of High-Income Users Considering Trip Distance and Trip Purpose

For short and medium trips, medium-income (MI) level users prefer motorcycle as well as public transport bus, human hauler, MRT, and BRT. But, with the increase of distance, they are shifted to MRT, BRT. Mode choice probability for medium-income level group people is shown in Figure 3. Car is not a preferred mode for a long trip (LT). In the case of a short and medium trip, the car is more preferable for other trips then business 
as well as commuting trips (CT).

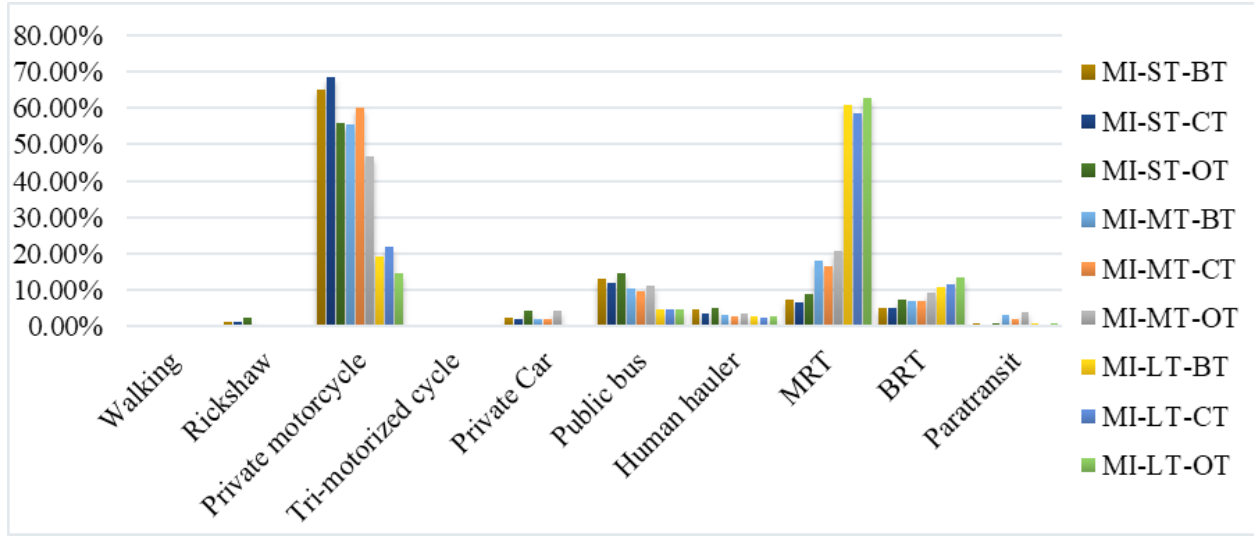

Fig. 3.

Mode Choice of Medium-Income Users Considering Trip Distance and Trip Purpose

The effect of trip distance and trip purpose on mode choice probability for low-income (LI) group people are shown in Figure 4. The probability of car mode choice is less than the motorcycle and other public transport. The motorcycle is more popular for short distance and popularity decreases with the increase of distance. The purpose is also important in this regard, for the business trip (BT) motor cycle is more preferable than commuting as well as other trips (OT). For long distance, MRT is more preferable than any other mode. With the increase of distance preference of BRT is also increase.

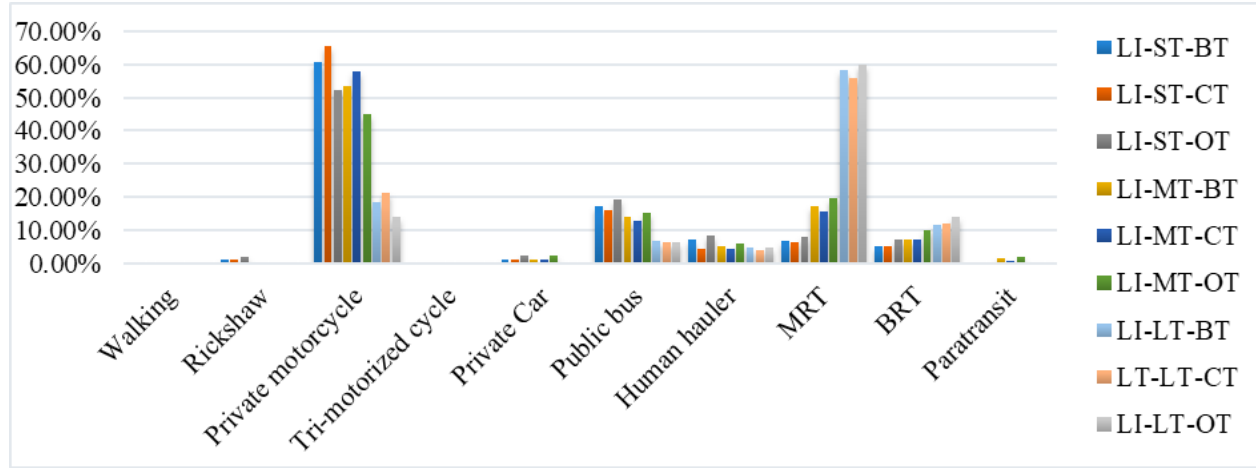

Fig. 4.

Mode Choice of Low-Income Users Considering Trip Distance and Trip Purpose

Total mode choice percentage considering all survey data is shown in Figure 5. Walking and Rickshaw is very low in this study. For long and medium distance their probability is almost zero considering trip purpose, trip length, and income level. Walking and rickshaw are $3 \%$ each from total mode share considering all survey data. From the analysis, it is found that the private motorcycle is more popular for short and medium distance. 
Because of its availability as well as travel with avoiding congestion. The motorcycle has a $15 \%$ mode share from all data.

Mode choice of the private car is comparatively higher for high-income level people. They also prefer a car for other trip purposes rather than business and comminuting trip. Car choice probability also decreases with the increase of trip distance. High-income people do not like to ride the public bus and human hauler. But their percentage increase for medium and low-income people. Public bus and human hauler are also preferable for short distance and medium trips than long distance trips.

From the analysis, MRT is more preferable for long distance travel. Considering a business trip, MRT is more preferable for the long trip than the short and medium trip. The same scenario is also found for commuting trips and other trips. Income level and trip purpose have a less significant effect on MRT mode choice. BRT is comparatively less preferable then MRT. But the trend of choice considering income level, trip purpose, and trip distance are almost similar to MRT.

It is found that the probability of paratransit is comparatively low. But the mode choice of the private motorcycle is large. In recent times, paratransit is increasing in the Dhaka city area. It will be a limitation of this study that due to confusing, survey responders mixed up with private motorcycle and paratransit mode. In this regard motorcycle cover a larger part of mode choice.

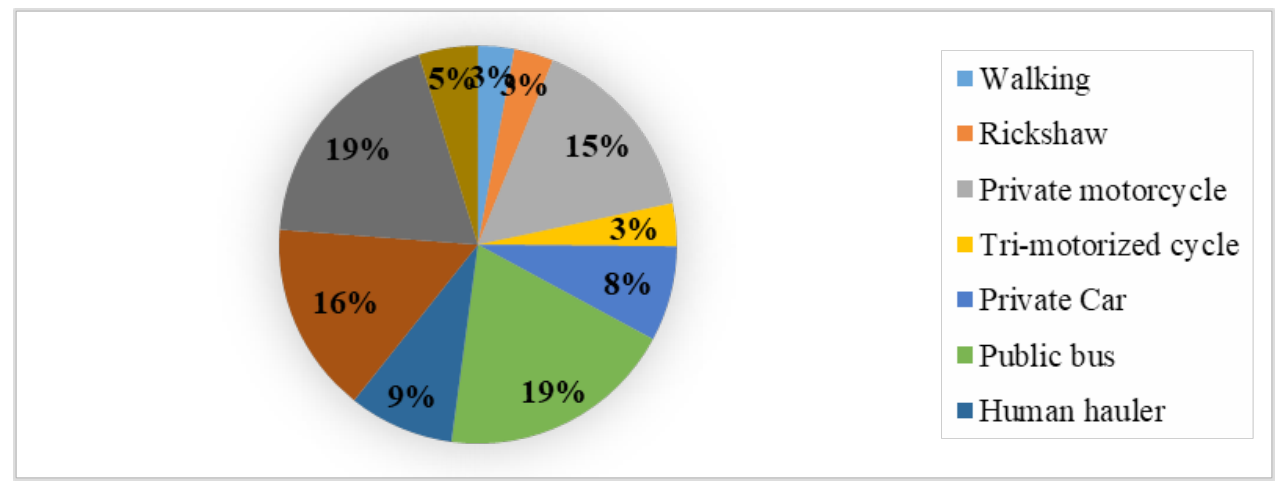

Fig. 5.

Modal Split Analysis

From Figure 6, it has been shown that MRT and BRT have more effect on travel distance than trip purpose and income level. For the short trip, MRT and BRT are preferable about $10 \%$ to $16 \%$. And it will be around $20 \%$ to $30 \%$ for the medium trip. In the case of the long trip, their percentage has changed in large amounts from $68 \%$ to $76 \%$. It is an indication that MRT and BRT can play significantly. 


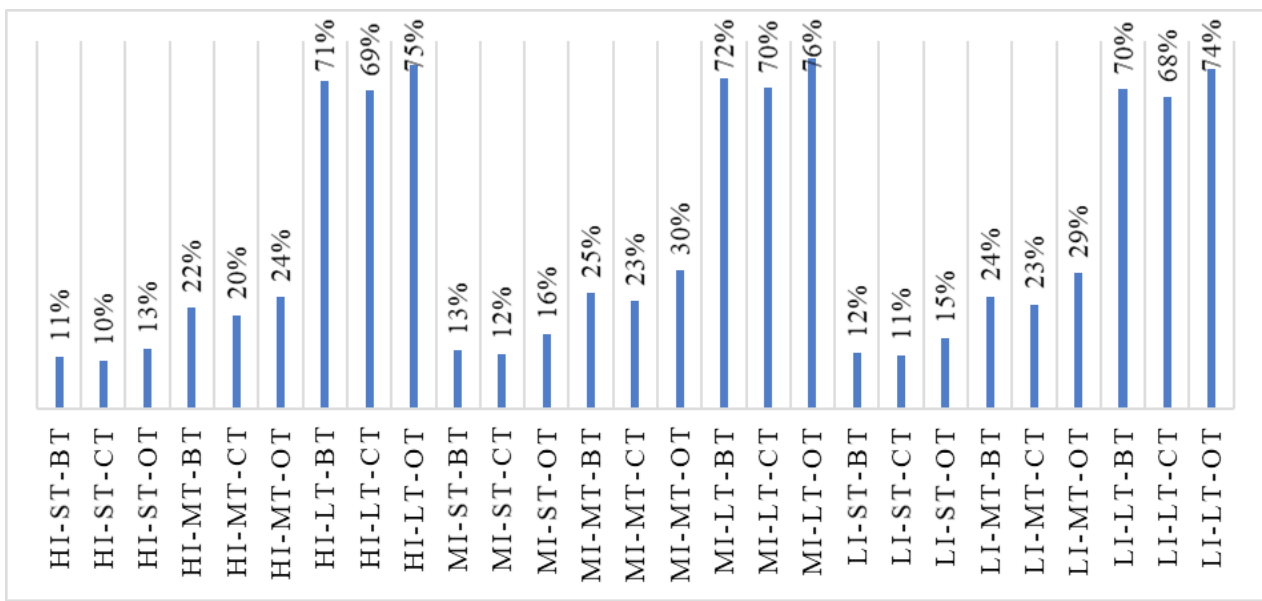

Fig. 6.

Combined Mode Choice Probability for MRT and BRT Considering Income Level, Trip Length and Trip Purpose

R-Square value are also calculated using different methods. All method indicates good analysis results which are shown in Table 7.

Table 7

R-Square Value for MNL Model

\begin{tabular}{|c|c|}
\hline Method Name & R-square Value \\
\hline Cox and Snell & 0.719 \\
\hline Nagelkerke & 0.761 \\
\hline McFadden & 0.438 \\
\hline
\end{tabular}

\section{Value of Time Estimation}

Value of travel time (VOT) is one of the significant parts of travel demand models and it is also important for management and appraisal of transport investment decisions. Value of time can be defined as the maximum amount of money that people are willing to sacrifice to save one unit of time, provide that all other trip related attributes remain constant. In simple linear models, the VOT is calculated as the ratio of parameter estimates related to travel time and travel cost considering all parameters are constants. In calculating VOT, both attributes to be used in the calculation must be found to be statically significant. Otherwise, no meaningful VOT can be calculated.

For estimation of the value of time, the binominal logit model is used in this study. Considering dependent variable as a preference (yes or no), coefficient of parameters of in-vehicle time, waiting time, cost, trip length, mode, seating option are estimated individually considering a combination of income level (high, low, medium) and trip purpose (business, comminuting, others). The alternatives differ on the following attributes: 
- In-vehicle travel time, IVT (min);

- Waiting Travel Time WT (min);

- Travel cost, TC (BDT);

- $\quad$ Seating option, SS;

- Travel length, TL (Km);

- Travel mode.

The utility function of the alternative has the form Eq. (3):

$U_{i}=\beta_{i w t} I V T+\beta_{w t} W T+\beta_{t c} T C+\beta_{s s} S S+\beta_{t l} T L+$ $+\beta_{m} M+\varepsilon$
Where, $\beta_{i w t}, \beta_{w t}, \beta_{t c}, \beta_{s s}, \beta_{t l}$ and $\beta_{m}$ are the coefficient of corresponded parameters and $\varepsilon$ is constant. Which are estimated from data. Then the value of in-vehicle time and value of waiting time are calculated by the following formula (Eq. (4) and Eq. (5)).

Value of in vehicle time $(\mathrm{VOT})=$ $\left(\beta_{i w t} / \beta_{t c}\right)^{*} 60 \mathrm{BDT} / \mathrm{hr}$

Value of Waiting Time (VOWT) = $\left(\beta_{w t} / \beta_{t c}\right)^{*} 60 \mathrm{BDT} / \mathrm{hr}$

\section{Table 8}

Result of Value of Time Considering Income Level and Trip Purpose

\begin{tabular}{|c|c|c|c|c|c|c|}
\hline \multirow{2}{*}{$\begin{array}{c}\text { Income level } \\
\text { and Trip } \\
\text { Purpose }\end{array}$} & \multirow{2}{*}{$\begin{array}{l}\text { Co-efficient } \\
\text { of in vehicle } \\
\text { time (min) }\end{array}$} & \multirow{2}{*}{$\begin{array}{c}\text { Co-efficient } \\
\text { of waiting } \\
\text { time (min) }\end{array}$} & \multirow{2}{*}{$\begin{array}{c}\text { Co- efficient } \\
\text { of cost }\end{array}$} & \multicolumn{2}{|c|}{ Value of time per hour } & \multirow{2}{*}{$\begin{array}{c}\text { Ratio of } \\
\text { (WT/IVT) }\end{array}$} \\
\hline & & & & $\begin{array}{c}\text { For In vehicle } \\
\text { time (IVT) }\end{array}$ & $\begin{array}{c}\text { For Waiting } \\
\text { time }(W T)\end{array}$ & \\
\hline HI_BT & -0.041 & -0.131 & -0.002 & 1115.75 & 3561.48 & 3.19 \\
\hline HI_CT & -0.045 & -0.207 & -0.006 & 480.21 & 2203.99 & 4.59 \\
\hline HI_OT & -0.027 & -0.099 & 0.004 & 443.33 & 1644.25 & 3.71 \\
\hline MI_BT & -0.026 & -0.086 & -0.015 & 101.63 & 335.06 & 3.29 \\
\hline MI_CT & -.0 .026 & -0.086 & -0.019 & 80.97 & 269.43 & 3.33 \\
\hline MI_OT & -0.026 & -0.042 & -0.012 & 133.93 & 211.03 & 1.58 \\
\hline LI_BT & -0.024 & -0.014 & -0.029 & 48.15 & 28.21 & 0.59 \\
\hline LI_CT & -0.022 & -0.027 & -0.037 & 36.39 & 44.79 & 1.23 \\
\hline LI_OT & -0.029 & 0.008 & -0.032 & 53.38 & 15.25 & 0.28 \\
\hline
\end{tabular}

The coefficient of in-vehicle time, waiting time and cost are shown in Table 8 which are got from nine individual analyses. Then the ratio of time to the cost is the value of time or in other words, the value of time in exchange for cost has been estimated. In-vehicle time, waiting time and ratio of waiting time to in-vehicle time are shown in Table 8 for considering segments of HI_BT, HI_CT,HI_OT, MI_BT, MI_CT, MI_OT, LI_BT, LI_CT, and LI_OT respectively. The coefficient of cost for high-income others trip and coefficient of waiting time for low-income other trips are found as positive. It can be interpreted that trips which are made by high-income people for others purpose are less concern about cost. Another noticeable fact that low-income people also do not worry about waiting time for other trip purposes.

Value of time represents a sequential considering important of trip purpose and income level. From Table 8, it is also found that high-income level people are more sensitive for waiting time than low-income level. Except for low-income level people, the ratio of waiting time (WT) and in-vehicle time (IVT) is found more than one. R-square values are shown in Table 9 which indicates the accuracy of this model. in this study, all $\mathrm{R}$-square value indicates good analysis result. 
Table 9

R-Square Value for Binary Logit Model

\begin{tabular}{|c|c|c|}
\hline Income Level and Trip Purpose & Cox and Snell R-square & Nagelkerke R-Square \\
\hline HI_BT & 0.166 & 0.228 \\
\hline HI_CT & 0.218 & 0.295 \\
\hline HI_OT & 0.122 & 0.166 \\
\hline MI_BT & 0.210 & 0.283 \\
\hline MI_CT & 0.204 & 0.279 \\
\hline MI_OT & 0.141 & 0.191 \\
\hline LI_BT & 0.210 & 0.289 \\
\hline LI_CT & 0.243 & 0.333 \\
\hline LI_OT & 0.239 & 0.325 \\
\hline
\end{tabular}

\section{Conclusion and Discussion}

In this Study, a mode choice model has been developed using SP data using a discrete choice model along with a question preparation technique that is discussed to represent the actual scenario in front of the trip makers considering exiting travel modes with un-exiting travel modes. Results indicate that mode choice is sensitive to income level, trip purpose and trip length along with characteristics and performance of each travel mode. Important attributes of mode choice of each mode also study. Lowincome and medium-income level people usually prefer BRT and MRT. This study reveals the most important features for a successful public transport system of MRT and BRT that can attract people especially for high-income people for long-distance travel. The value of time is also calculated according to income level and trip purpose. Waiting time shows always higher value than in-vehicle time. The ratio of waiting time and in-vehicle time maintains a sequential relationship for trip purpose and income level of the trip maker. Mode choice behavior of Dhaka city dwellers has been represented in this paper considering income level, trip purpose, and trip length. Mode choice behavior and value of time results can play a vital role in policy-making on Dhaka city transportation planning.

\section{Acknowledgements}

Data are collected from NKDOS consortium (NK-OCG-SYSTRA-DMRC-NKI-DDC). Project name: Consultancy services for detailed design and tender assistance for Dhaka Mass Rapid Transit Development (MRT Line-1).

\section{References}

Ben-Akiva, M.E.; Lerman, S.R. 1985. Discrete Choice Analysis: Theory and Application to Travel Demand. MIT Press. $412 \mathrm{p}$.

DITS. 1993. Greater Dhaka Metropolitan Area Integrated Transport Study, Prepared by PPK Consultants Declan International and Development Design Consultant (DDC), Dhaka.

Enam, A. 2010. Developing a Comprehensive Mode Choice to Capture the Preferences for Mass Rapid Transit in Dhaka. (M.Sc. Thesis), Department of Civil Engineering, Bangladesh University of Engineering \& Technology, Dhaka. 130p. 
FS MRT line-1. 2018. The preparatory study on the Dhaka mass rapid transit development project (line 1) in Bangladesh, prepared by JICA, ALMEC Corporation, Oriental Consultants Global Co., Ltd, Nippon Koei Co., Kathahira \& Engineers International.

Hasan, S. 2007. Development of a Travel Demand Model for Dhaka City. (M.Sc. Thesis), Department of Civil Engineering, Bangladesh University of Engineering \& Technology, Dhaka.

Koppelman, F. S.; Bhat, C. 2006. A SelfInteresting Course in mode Choice Modeling: Multinomial and nested Logit Model. Federal Transit Administration. Washington, DC. USA. 241p.
Ortuzar, J. D.D.; Willumsen, L.G. 2011. Modelling Transport, $4^{\text {th }}$ Edition. John Willey \& Sons, New York. USA. 607 p.

RSTP. 2016. Revision and updating of the strategic transportation plan for Dhaka, Prepared by ALMEC Corporation, Oriental Consultants Global Co. Ltd, Kathahira \& Engineers International.

STP. 2005. Strategic Transport Plan for Dhaka. Louis Berger Group and Bangladesh Consultant Ltd.

Svenson, O. 1979. Process Descriptions of Decision making, Organizational behavior and human performance 23(1): 86-112. 